Pacific

Journal of

Mathematics

\title{
UNIQUENESS OF SHALIKA FUNCTIONALS: THE ARCHIMEDEAN CASE
}

Avraham Aizenbud, Dmitry Gourevitch ANd Hervé JaCQuet 


\title{
UNIQUENESS OF SHALIKA FUNCTIONALS: THE ARCHIMEDEAN CASE
}

\author{
Avraham Aizenbud, DMitry Gourevitch ANd HeRvé JACQUet
}

Let $F$ be either $\mathbb{R}$ or $\mathbb{C}$. Let $(\pi, V)$ be an irreducible admissible smooth Fréchet representation of $\mathrm{GL}_{2 n}(F)$. A Shalika functional $\phi: V \rightarrow \mathbb{C}$ is a continuous linear functional such that for any $g \in \mathbf{G L}_{n}(F), A \in \operatorname{Mat}_{n \times n}(F)$ and $v \in V$ we have

$$
\phi\left[\pi\left(\begin{array}{ll}
g & A \\
0 & g
\end{array}\right) v\right]=\exp \left(2 \pi i \operatorname{Re} \operatorname{Tr}\left(g^{-1} A\right)\right) \phi(v) .
$$

In this paper we prove that the space of Shalika functionals on $V$ is at most one-dimensional.

For nonarchimedean $F$ (of characteristic zero) this theorem was proved by Jacquet and Rallis.

\section{Introduction}

Let $F$ be either $\mathbb{R}$ or $\mathbb{C}$. Let $(\pi, V)$ be an admissible smooth Fréchet representation of $\mathrm{GL}_{2 n}(F)$. We assume that $V$ is the canonical completion of an irreducible Harish-Chandra $(\mathfrak{g}, K)$ - module in the sense of Casselman and Wallach [Wallach 1992, Chapter 11]. A Shalika functional $\phi: V \rightarrow \mathbb{C}$ is a continuous linear functional such that for any $g \in \mathrm{GL}_{n}(F), A \in \operatorname{Mat}_{n \times n}(F)$ and $v \in V$ we have

$$
\phi\left[\pi\left(\begin{array}{ll}
g & A \\
0 & g
\end{array}\right) v\right]=\exp \left(2 \pi i \operatorname{Re}\left(\operatorname{Tr}\left(g^{-1} A\right)\right)\right) \phi(v) .
$$

In this paper we prove the following theorem.

Theorem 1.1. If $(\pi, V)$ is an irreducible admissible smooth Fréchet representation of $\mathrm{GL}_{2 n}(F)$, the space of Shalika functionals on $V$ is at most one-dimensional.

For nonarchimedean $F$ of characteristic zero this theorem was proved in [Jacquet and Rallis 1996]. The proof there is based on the fact (also proved in the same paper) that $\left(\mathrm{GL}_{2 n}(F), \mathrm{GL}_{n}(F) \times \mathrm{GL}_{n}(F)\right)$ is a Gelfand pair, and on the method of [Friedberg and Jacquet 1993, Section 3] of integration of Shalika functionals.

MSC2000: 22E45.

Keywords: multiplicity one, Gelfand pairs, Shalika functionals, uniqueness of linear periods. Aizenbud and Gourevitch were partially supported by a BSF grant, a GIF grant, and an ISF Center of Excellency grant. Aizendbud was also partially supported by ISF grant No. 583/09. 
In the archimedean case those two ingredients also exist: the argument in [Friedberg and Jacquet 1993, Section 3] is also valid in this case, and the fact that $\left(\mathrm{GL}_{2 n}(F), \mathrm{GL}_{n}(F) \times \mathrm{GL}_{n}(F)\right)$ is a Gelfand pair is shown in [Aizenbud and Gourevitch 2009].

The proof we present here is similar to that in [Jacquet and Rallis 1996]. The main difference is that we have to prove the continuity of a certain linear form.

Structure of the proof. We construct a linear map from the space of Shalika functionals to the space of linear periods - that is, linear functionals on $V$ invariant under $\mathrm{GL}_{n}(F) \times \mathrm{GL}_{n}(F)$ - and prove this map's injectivity. Hence uniqueness of linear periods implies uniqueness of Shalika functionals. The uniqueness of linear periods amounts to the fact, already mentioned, that $\operatorname{GL}_{2 n}(F), \mathrm{GL}_{n}(F) \times \mathrm{GL}_{n}(F)$ ) is a Gelfand pair.

Structure of the paper. Section 2 fixes notation and terminology. In Section 3 we describe a way of obtaining a linear period from a Shalika functional by integration, as in [Friedberg and Jacquet 1993, Section 3]. In Section 4 we investigate the properties of the obtained period. In Section 5 we explain how this implies the uniqueness of Shalika functionals.

\section{Notation and preliminaries}

- Henceforth we fix an archimedean field $F$ (that is, $F$ is $\mathbb{R}$ or $\mathbb{C}$ ).

- For a group $G$ acting on a vector space $V$ we denote by $V^{G}$ the space of $G$ invariant vectors in $V$. For a character $\chi$ of $G$ we denote by $V^{G, \chi}$ the space of $(G, \chi)$-equivariant vectors in $V$.

- For a smooth real algebraic variety $M$ we denote by $\mathscr{S}(M)$ the space of Schwartz functions on $M$, that is, the space of smooth functions that are rapidly decreasing as well as all their derivatives. For precise definition see, for example, [Aizenbud and Gourevitch 2008].

- We fix a natural number $n$ and write $G:=\operatorname{GL}_{2 n}(F)$.

- We fix a norm on $G$ by

$$
\|g\|:=\sum_{1 \leq i, j \leq 2 n}\left|g_{i j}\right|^{2}+\sum_{1 \leq i, j \leq 2 n}\left|\left(g^{-1}\right)_{i j}\right|^{2} .
$$

- We set $G_{1}:=\left\{\left(\begin{array}{cc}g & 0 \\ 0 & \mathrm{Id}\end{array}\right) \mid g \in \mathrm{GL}_{n}(F)\right\} \subset G$.

- We denote by $v: \mathrm{GL}_{n}(F) \rightarrow G_{1}$ the isomorphism defined by $v(g):=\left(\begin{array}{ll}g & 0 \\ 0 & \mathrm{Id}\end{array}\right)$. 
Note that for any $X \in \operatorname{Mat}(n \times n, F)$, we have $d v(X)=\left(\begin{array}{cc}X & 0 \\ 0 & 0\end{array}\right)$.

- We set $H:=\left\{\left(\begin{array}{ll}g & 0 \\ 0 & g\end{array}\right) \mid g \in \mathrm{GL}_{n}(F)\right\} \subset G$.

- We set $U:=\left\{\left(\begin{array}{cc}\mathrm{Id} & A \\ 0 & \mathrm{Id}\end{array}\right) \mid A \in \operatorname{Mat}_{n \times n}(F)\right\} \subset G$.

- We denote by $\mu: \operatorname{Mat}(n \times n, F) \rightarrow U$ the isomorphism defined by

$$
\mu(A):=\left(\begin{array}{cc}
\mathrm{Id} & A \\
0 & \mathrm{Id}
\end{array}\right) .
$$

Note that for any $X \in \operatorname{Mat}(n \times n, F)$, we have $d \mu(X)=\left(\begin{array}{ll}0 & X \\ 0 & 0\end{array}\right)$.

- We define a homomorphism $\tau: U \rightarrow F$ by

$$
\tau(\mu(A)):=\operatorname{Tr}(A) .
$$

- We let $\psi$ be the additive character of $F$ defined by $\psi(x):=e^{2 \pi \mathrm{i} \operatorname{Re} x}$. We define an homomorphism $\Psi: U \rightarrow F^{\times}$by

$$
\Psi:=\psi \circ \tau \text {. }
$$

We extend $\Psi$ to an homomorphism $\Psi: H U \rightarrow F^{\times}$trivial on $H$.

- We denote by $K$ the standard maximal compact subgroup of $G$. Thus $K=$ $O(2 n)$ if $F=\mathbb{R}$ and $K=U(2 n)$ if $F=\mathbb{C}$.

Admissible representations. We consider admissible smooth Fréchet representations of $G$, that is, smooth admissible representations $(\pi, V)$ of $G$ such that $V$ is a Fréchet space and, for any continuous seminorm $\alpha$ on $V$, there exists another continuous seminorm $\beta$ on $V$ and a natural number $M$ such that for any $g \in G$,

$$
\alpha(\pi(g) v) \leq \beta(v)\|g\|^{M} .
$$

By the Casselman-Wallach theorem [Wallach 1992, Chapter 11], $V$ may be regarded as the canonical model of an irreducible Harish-Chandra $(\mathfrak{g}, K)$-module. By the Casselman embedding theorem [Casselman 1980], $V$ can be realized as a closed subspace of a principal series representation. We denote by $\widetilde{V}$ the canonical model of the contragredient Harish-Chandra $(\mathfrak{g}, K)$-module. It is a subspace of the topological dual $V^{*}$ of $V$.

\section{Integration of Shalika functionals}

In this section we fix:

- an irreducible admissible smooth Fréchet representation $(\pi, V)$ of $G$;

- a Shalika functional $\lambda$ on $V$, that is, $\lambda \in\left(V^{*}\right)^{H U, \Psi}$. 
Theorem 3.1. There exists $M \in \mathbb{R}$ such that for any $v \in V$ and over the region of $s \in \mathbb{C}$ with $\operatorname{Re} s>M$, the integral

$$
L_{\lambda, v}(s):=\int_{g \in G_{1}} \lambda(\pi(g) v)|\operatorname{det} g|^{s-1 / 2} d g
$$

converges absolutely and is a holomorphic function of $s$.

Moreover, $L_{\lambda, v}(s)$ has meromorphic continuation to the complex plane and is a holomorphic multiple of the $L$-function $L_{\pi}$ of the representation $\pi$. Finally, for any $\lambda \neq 0$ there exists $v \in V$ such that $L_{\lambda, v}=L_{\pi}$.

In [Friedberg and Jacquet 1993, Proposition 3.1] this theorem is proved under the assumption that there exists a continuous seminorm $\beta$ on $V$ satisfying $|\lambda(\pi(g) v)| \leq$ $\beta(v)$ for any $g \in G$.

This may not be true in general. However:

Lemma 3.2. There exist $M>0$ and a continuous seminorm $\beta$ on $V$ such that $|\lambda(\pi(g) v)| \leq|\operatorname{det} g|^{-M} \beta(v)$ for any $g \in G_{1}$.

Before proving the lemma, we check that, with its help, the proof of Theorem 3.1 is still valid. To that end, we first prove the following lemma, which is an analog of [Friedberg and Jacquet 1993, Lemma 3.1].

Lemma 3.3. There is a continuous seminorm $\gamma$ on $V$ such that, for any $v \in V$,

$$
|\lambda(\pi(g) v)| \leq\left|\operatorname{det} b^{-1} a\right|^{-M} \gamma(v) \quad \text { for } g=u\left(\begin{array}{ll}
a & 0 \\
0 & b
\end{array}\right) k,
$$

with $a, b \in \mathrm{GL}(n, F), u \in U, k \in K$. For any $v \in V$, there is $\Phi_{v} \in \mathscr{Y}(\operatorname{Mat}(n \times n, F))$ such that

$$
|\lambda(\pi(g) v)| \leq \Phi\left(b^{-1} a\right)\left|\operatorname{det} b^{-1} a\right|^{-M},
$$

for $g$ of the form given in $(*)$.

Proof. For the first assertion, we have $\lambda(\pi(g) v)=\Psi(u) \lambda\left(\pi\left(v\left(b^{-1} a\right)\right) \pi(k) v\right)$. Hence

$$
|\lambda(\pi(g) v)| \leq\left|\operatorname{det} b^{-1} a\right|^{-M} \beta(\pi(k) v) .
$$

There is another continuous seminorm $\gamma$ such that, for all $k \in K$,

$$
\beta(\pi(k) v) \leq \gamma(v) .
$$

The first assertion follows.

For the second assertion, we go through the proof of [Friedberg and Jacquet 1993, Lemma 3.1] (which is the estimate above with $M=0$ ) and arrive at once at the present estimate. 
The proof of Theorem 3.1 is still valid. The only modification is that we need to check that, under our weaker assumption, two integrals in [Friedberg and Jacquet 1993] which depend on $s \in \mathbb{C}$, are still absolutely convergent for $\operatorname{Re} s \gg 0$.

The first integral is integral [Friedberg and Jacquet 1993, 45]:

$$
\int \lambda(\pi(g) v) \Phi(g)|\operatorname{det} g|^{s+n-1 / 2} d^{\times} g,
$$

where $\Phi \in \mathscr{S}(\operatorname{Mat}(2 n \times 2 n, F))$. We write

$$
g=\left(\begin{array}{ll}
a & x \\
0 & b
\end{array}\right) k
$$

Then

$$
d^{\times} g=|\operatorname{det} a|^{-n} d^{\times} a d^{\times} b d x d k .
$$

By Lemma 3.3, the integral of the absolute value is bounded by

$$
\int|\operatorname{det} a|^{\operatorname{Re} s-M-1 / 2}|\operatorname{det} b|^{\operatorname{Re} s+M+n-1 / 2}\left|\Phi\left[\left(\begin{array}{ll}
a & x \\
0 & b
\end{array}\right) k\right]\right| d^{\times} a d^{\times} b d x d k .
$$

This does converge absolutely for $\operatorname{Re} s \gg 0$.

The second integral is integral [Friedberg and Jacquet 1993, 48]. It has the form

$$
\int \lambda\left[\pi\left(\begin{array}{cc}
a & 0 \\
0 & \mathrm{Id}
\end{array}\right) \pi(x) v\right]|\operatorname{det} a|^{s-1 / 2} d^{\times} a d \mu(x),
$$

where $\mu$ is the measure on $\operatorname{SL}(2 n, F)$ defined by

$\int f(x) d \mu(x)=\int f\left[\left(\begin{array}{cc}b^{-1} & 0 \\ 0 & \operatorname{Id}\end{array}\right)\left(\begin{array}{cc}\operatorname{Id} & u \\ 0 & \operatorname{Id}\end{array}\right)\left(\begin{array}{cc}\operatorname{Id} & 0 \\ 0 & b\end{array}\right) k\right] \Upsilon\left(u, b^{-1}, b ; k\right)|\operatorname{det} b|^{n} d^{\times} b d u d k$.

In this formula $k$ is integrated over $K^{\prime}=K \cap \operatorname{SL}(2 n, F)$ and the function $\Upsilon$ is in $\mathscr{S}\left(\operatorname{Mat}(n \times n, F)^{3} \times K^{\prime}\right)$. The integral of the absolute value of the integrand is bounded by

$\int \Phi_{v}\left(a b^{-2}\right)\left|\operatorname{det} a b^{-2}\right|^{-M}|\Upsilon|\left(u, b^{-1}, b ; k\right)|\operatorname{det} b|^{n} d^{\times} b d u d k|\operatorname{det} a|^{\operatorname{Re} s-1 / 2} d^{\times} a$.

After changing $a$ to $a b^{2}$, the integral decomposes into a product:

$$
\int \Phi_{v}(a)|\operatorname{det} a|^{\operatorname{Re} s-M-1 / 2} d^{\times} a \times \int|\Upsilon|\left(u, b^{-1}, b ; k\right)|\operatorname{det} b|^{n+2 \operatorname{Re} s-1} d^{\times} b d u d k .
$$

The first integral converges for $\operatorname{Re} s \gg 0$. The second integral converges for all $s$. It remains to prove Lemma 3.2. We will prove something more general:

Lemma 3.4. There exists $M_{0}>0$ such that, for any polynomial $P$ on the real vector space $\operatorname{Mat}(n \times n, F)$, there exists a continuous seminorm $\beta_{P}$ on $V$ such that 
for any $g \in \mathrm{GL}_{n}(F)$ we have

$$
|\lambda(\pi(v(g)) v)| \leq \beta_{P}(v) \frac{1}{|P(g)|}|\operatorname{det} g|^{-M_{0}} .
$$

Proof. We have $\lambda(\pi(\mu(X)) v)=\psi(\operatorname{Tr} X) \lambda(v)$ for all $X \in \operatorname{Mat}(n \times n, F)$. Hence

$$
\lambda(d \pi(d \mu(X)) v)=2 \pi \mathrm{i} \operatorname{Re}(\operatorname{Tr} X) \lambda(v) \quad \text { for all } X \in \operatorname{Mat}(n \times n, F),
$$

which implies

$\lambda(\pi(v(g)) d \pi(d \mu(X)) v)=2 \pi \mathrm{i} \operatorname{Re} \operatorname{Tr}(g X) \lambda(\pi(v(g)) v)$

for all $X \in \operatorname{Mat}(n \times n, F)$ and $g \in \mathrm{GL}_{n}(F)$.

Similarly, if $Q$ is a polynomial on the real vector space $\operatorname{Mat}(n \times n, F)$, there is an element $X_{Q}$ of the enveloping algebra of $\mathfrak{g l}_{2 n}(F)$ such that

$$
\lambda\left(\pi(v(g)) d \pi\left(X_{Q}\right) v\right)=Q(g) \lambda(\pi(v(g)) v) \quad \text { for all } g \in \mathrm{GL}_{n}(F) .
$$

We know that there exist a continuous seminorm $\beta$ on $V$ and a natural number $M$ such that $|\lambda(\pi(g) v)| \leq \beta(v)\|g\|^{M}$ for any $g \in G$. Therefore for any $g \in \mathrm{GL}_{n}(F)$ we have

$$
|Q(g) \lambda(\pi(v(g)) v)|=\left|\lambda\left(\pi(v(g)) d \pi\left(\mu\left(X_{Q}\right)\right) v\right)\right| \leq \beta\left(d \pi\left(X_{Q}\right) v\right)\|v(g)\|^{M} .
$$

Note that $\|v(g)\|^{M}=P_{0}(g)|\operatorname{det} g|^{-2 M}$ for a suitable polynomial $P_{0}$ on the real vector space $\operatorname{Mat}(n \times n, F)$. Therefore, we have, with $M_{0}=2 M$,

$$
|\lambda(\pi(v(g)) v)| \leq \beta\left(d \pi\left(X_{Q}\right) v\right) \frac{P_{0}(g)}{|Q(g)|}|\operatorname{det} g|^{-M_{0}} .
$$

We may take $Q$ of the form $Q=P_{0} P$, where $P$ is another polynomial. Since $v \mapsto \beta\left(d \pi\left(X_{Q}\right) v\right)$ is a continuous seminorm, the lemma follows.

\section{Properties of $L_{\lambda, v}$}

Theorem 4.1. Let $(\pi, V)$ be an irreducible admissible smooth Fréchet representation of $G$. Fix a Shalika functional $\lambda \in\left(V^{*}\right)^{H U, \Psi}$ and a vector $v \in V$. For any polynomial $p$, the product $p(s) L_{\lambda, v}(s)$ is bounded at infinity on every vertical strip of finite width.

This is an immediate consequence of the next two results:

Lemma 4.2 [Friedberg and Jacquet 1993, Section 3.3]. For $\operatorname{Re}(s)$ large enough, $L_{\lambda, v}(s)$ is a finite sum of functions of the type

$$
\mathscr{L}_{u, \xi, \Phi}(s):=\int_{g \in G} \Phi(g) \xi(\pi(g) u)|\operatorname{det} g|^{s+n-1 / 2} d g,
$$

where $\Phi \in \mathscr{S}(\operatorname{Mat}(2 n \times 2 n, F)), u \in V, \xi \in V^{*}$. 
Theorem 4.3. Let $(\pi, V)$ be an irreducible admissible smooth Fréchet representation of $G$. Let $\Phi \in \mathscr{S}(\operatorname{Mat}(2 n \times 2 n, F)), u \in V$ and $\xi \in \widetilde{V}$. Then $\mathscr{L}_{u, \xi, \Phi}(s)$ has a meromorphic continuation to $\mathbb{C}$ whose product by any polynomial is bounded at infinity on any vertical strip. The continuation is a holomorphic multiple of $L_{\pi}(s)=L(s, \pi)$. It satisfies the functional equation

$$
\int \widehat{\Phi}(g) \xi\left(\pi\left(^{t} g^{-1}\right) u\right)|\operatorname{det} g|^{1-s+n-1 / 2} d g=\gamma(s, \pi, \psi) \mathscr{L}_{u, \xi, \Phi}(s)
$$

where $\gamma(s, \pi, \psi):=\varepsilon(s, \pi, \psi) \frac{L(1-s, \tilde{\pi})}{L(s, \pi)}$ and

$$
\widehat{\Phi}(X):=\int_{\operatorname{Mat}(2 n \times 2 n, F)} \Phi(Y) \psi\left(\operatorname{tr}\left(X Y^{t}\right)\right) d Y .
$$

These assertions remain true if $\xi$ is in $V^{*}$, the topological dual of $V$.

This theorem is proved in [Godement and Jacquet 1972] in slightly narrower generality: the vectors $u$ and $\xi$ are $K$-finite and the function $\Phi$ is the product of a Gaussian function and a polynomial. For the reader's convenience we indicate how to extend the result to our context. We need the following lemma.

Lemma 4.4. Let $T \subset G$ be the torus of diagonal matrices. We will also regard $T$ as the subset $\left(F^{\times}\right)^{2 n}$ of $F^{2 n}$. Let $\chi: T \rightarrow \mathbb{C}^{\times}$be a multiplicative character. Let $(\pi, V)$ be the corresponding representation of principal series of $G$. Let $v \in V$ and $\xi \in \widetilde{V}$. Let $\Phi$ be a Schwartz function on $\operatorname{Mat}(2 n \times 2 n, F)$.

Then there exists a Schwartz function $\phi \in \mathscr{S}\left(F^{2 n}\right)$ such that

$$
\int_{g \in G} \Phi(g) \xi(\pi(g) v)|\operatorname{det} g|^{s+n-1 / 2} d g=\int_{t \in T} \phi(t) \chi(t)|\operatorname{det} t|^{s} d t
$$

for any $s \in \mathbb{C}$ such that the integral on the right converges absolutely.

Proof. Let $N$ denote the group of upper triangular matrices with unit diagonal. Set $B=T N$ and let $\delta_{B}$ be the module of the group $B$. Realize $V$ as the space of smooth functions on $G$ that satisfy $f(t g)=\chi(t) f(g) \delta_{B}^{1 / 2}(t)$ and $f(u g)=f(g)$ for any $t \in T$ and $u \in N$. Realize also $\widetilde{V}$ in the corresponding way. Then

$$
\xi(\pi(g) v)=\int_{k \in K} v(k g) \xi(k) d k,
$$

where $K$ is the standard maximal compact subgroup. Now

$$
\begin{array}{rl}
\int_{G} \Phi(g) \xi(\pi(g) v)|\operatorname{det} g|^{s+(n-1) / 2} & d g \\
& =\int_{G} \int_{K} \Phi(g) v(k g) \xi(k)|\operatorname{det} g|^{s(n-1) / 2} d g d k \\
& =\int_{G} \int_{K} \Phi\left(k^{-1} g\right) v(g) \xi(k)|\operatorname{det} g|^{s+(n-1) / 2} d g d k .
\end{array}
$$


To compute this integral we set

$$
g=\left(\begin{array}{cccc}
a_{1} & u_{1,2} & \cdots & u_{1,2 n} \\
0 & a_{2} & \cdots & u_{2,2 n} \\
& & \ddots & \\
0 & 0 & \cdots & a_{2 n}
\end{array}\right) k^{\prime}
$$

Then

$$
d g=\left|a_{1}\right|^{1-2 n}\left|a_{2}\right|^{2-2 n} \cdots\left|a_{2 n-1}\right|^{-1} \otimes d^{\times} a_{i} \otimes d u_{i, j} d k^{\prime} .
$$

We set

$$
\phi\left(a_{1}, \ldots, a_{2 n}\right):=\int \Phi\left[k^{-1}\left(\begin{array}{cccc}
a_{1} & u_{1,2} & \cdots & u_{1,2 n} \\
0 & a_{2} & \cdots & u_{2,2 n} \\
& & \ddots & \\
0 & 0 & \cdots & a_{2 n}
\end{array}\right) k^{\prime}\right] v\left(k^{\prime}\right) \xi(k) d k d k^{\prime} \otimes d u_{i, j} .
$$

Clearly $\phi$ is a Schwartz function on $F^{2 n}$ and

$$
\int_{g \in G} \Phi(g) \xi(\pi(g) v)|\operatorname{det} g|^{s+(n-1) / 2} d g=\int_{t \in T} \phi(t) \chi(t)|\operatorname{det} t|^{s} d t
$$

for any $s \in \mathbb{C}$ such that the integral on the right converges.

Proof of Theorem 4.3. The representation $(\pi, V)$ is a subrepresentation of a principal series representation determined by a character $\chi$ of $T$ and the representation $(\tilde{\pi}, \widetilde{V})$ is then a quotient of the representation determined by $\chi^{-1}$. For $u \in V$ and $\xi \in \widetilde{V}$ (or $\xi$ in the principal series determined by $\chi^{-1}$ ) we have

$$
\begin{aligned}
& \mathscr{L}_{u, \xi, \Phi}(s) \\
& =\int_{\left(a_{1}, \ldots, a_{2 n}\right) \in F^{\times 2 n}} \phi\left(a_{1}, a_{2}, \ldots, a_{2 n}\right) \chi_{1}\left(a_{1}\right)\left|a_{1}\right|^{s} \ldots \chi_{2 n}\left(a_{2 n}\right)\left|a_{2 n}\right|^{s} d^{\times} a_{1} \ldots d^{\times} a_{2 n} .
\end{aligned}
$$

The right side extends to a meromorphic function of $s$ and the product of this function by any polynomial is bounded at infinity in any vertical strip. Moreover, the function $\phi$ depends continuously on $\Phi, u \in V, \xi \in \widetilde{V}$. Therefore the analytic continuation depends continuously on $\Phi, v \in V, \xi \in \widetilde{V}$. By continuity, it has the properties stated in the Theorem. To extend further to the case where $\xi$ is in the topological dual $V^{*}$ we appeal to the Dixmier-Malliavin Lemma [1978] applied to the representation of $\mathrm{SL}_{2 n}(F)$ on $\mathscr{S}\left(\operatorname{Mat}_{2 n \times 2 n}(F)\right)$ defined by

$$
g_{1} \Phi(X):=\Phi\left(g_{1}^{-1} X\right) .
$$

Thus we may assume $\Phi$ is of the form

$$
\Phi(X)=\int_{\mathrm{SL}_{2 n}(F)} \Phi_{1}\left(g_{1}^{-1} X\right) f\left(g_{1}\right) d g_{1},
$$


where $f_{1}$ is a $C^{\infty}$ function of compact support on $\operatorname{SL}_{2 n}(F)$. Then

$$
\mathscr{L}_{u, \xi, \Phi}=\mathscr{L}_{u, \xi_{1}, \Phi_{1}}, \quad \text { where } \quad \xi_{1}(v):=\xi\left(\pi\left(f_{1}\right) v\right) .
$$

Now $\xi_{1}$ is in $\widetilde{V}$ and our assertion follows.

Remark 4.5. The previous result with $\xi \in V^{*}$ is used without comment in formula (57) of [Friedberg and Jacquet 1993]. This is why we included a sketch of the proof.

Theorem 4.6. There exists $M>0$ such that, for any even integer $M^{\prime} \geq 2$ and any polynomial $p$ on $\mathbb{C}$, there exists a seminorm $\beta$ on $V$ such that

$$
\left|p L_{\lambda, v}\right|_{M^{\prime}+M+\mathrm{i} \mathbb{R}} \mid \leq \beta(v) .
$$

Lemma 4.7. There exists $M>0$ such that, for any even integer $M^{\prime} \geq 2$, there exists a continuous seminorm $\beta$ on $V$ such that $\left|L_{\lambda, v}(s)\right| \leq \beta(v)$ for $\operatorname{Re} s=M^{\prime}+M$.

Proof. From Lemma 3.4 we know that there exists $M_{0}>0$ and for any polynomial $P$ on $\operatorname{Mat}(n \times n, F)$ a continuous seminorm $\beta_{P}$ such that

$$
\lambda(\pi(v(g)) v) \leq \beta_{P}(v) \frac{1}{|P(g)|}|\operatorname{det} g|^{-M_{0}} .
$$

Let $M:=\frac{1}{2}+n^{2}+M_{0}$. Let $M^{\prime} \geq 2$ be an even integer and let $s \in \mathbb{C}$ with $\operatorname{Re} s=$ $M+M^{\prime}$. Let

$$
P(X)=|\operatorname{det} X|^{M^{\prime}} \prod_{i, j}\left(1+X_{i j} \bar{X}_{i j}\right)
$$

Let

$$
\beta(v):=\beta_{P}(v) \int_{X \in \operatorname{Mat}(n \times n, F)} \frac{d X}{\prod_{i, j}\left(1+X_{i j} \bar{X}_{i j}\right)} .
$$

Now

$$
\begin{aligned}
\left|L_{\lambda, v}\right| & =\left.\left|\int_{\mathrm{GL}_{n}(F)} \lambda(\pi(v(g)) v)\right| \operatorname{det} g\right|^{s-1 / 2} d g \mid \\
& \leq \int_{\mathrm{GL}_{n}(F)} \beta_{P}(v) \frac{1}{|P(g)|}|\operatorname{det} g|^{-M_{0}}|\operatorname{det} g|^{n^{2}+M^{\prime}+M_{0}} d g \\
& =\int_{\mathrm{GL}_{n}(F)} \beta_{P}(v) \frac{1}{|P(g)|}|\operatorname{det} g|^{n^{2}+M^{\prime}} d g \\
& =\int_{X \in \operatorname{Mat}(n \times n, F)} \beta_{P}(v) \frac{1}{|P(X)|}|\operatorname{det} X|^{M^{\prime}} d X=\beta(v) .
\end{aligned}
$$

Proof of Theorem 4.6. For any $g \in \mathrm{GL}_{n}(F)$ we have

$$
L_{\lambda, \pi(\nu(g)) v}(s)=|\operatorname{det} g|^{1 / 2-s} L_{\lambda, v}(s) .
$$


We can apply this to $g=\exp (t X)$, with $t \in \mathbb{R}$ and $X \in \operatorname{Mat}(n \times n, F)$. We get

$$
L_{\lambda, \pi(v(g)) v}(s)=|\operatorname{det} \exp (t X)|^{1 / 2-s} L_{\lambda, v}(s) .
$$

Differentiating with respect to $t$ at $t=0$, we get

$$
L_{\lambda, d \pi(d v(X)) v}(s)=\left(\frac{1}{2}-s\right) c(X) L_{\lambda, v}(s),
$$

where $c(X)=\operatorname{Tr} X$ if $F=\mathbb{R}$ and $c(X)=2 \operatorname{Re} \operatorname{Tr} X$ if $F=\mathbb{C}$. Similarly, for any polynomial $p$ on $\mathbb{C}$ there exists $X_{p}$ in the universal enveloping algebra of $\mathfrak{g l}_{2 n}(F)$ such that

$$
L_{\lambda, d \pi\left(X_{p}\right) v}(s)=p(s) L_{\lambda, v}(s) .
$$

The theorem follows now from Lemma 4.7.

Notation 4.8. Define another representation $\pi^{\theta}$ on the same space $V$ by $\pi^{\theta}(g):=$ $\pi\left(\left(g^{t}\right)^{-1}\right)$. Recall that $\pi^{\theta} \cong \tilde{\pi}$.

For any Shalika functional $\lambda: \pi \rightarrow \mathbb{C}$ we define $\lambda^{\theta}: \pi^{\theta} \rightarrow \mathbb{C}$ by

$$
\lambda^{\theta}(v):=\lambda\left(\pi\left(\begin{array}{cc}
0_{n n} & \operatorname{Id}_{n n} \\
-\operatorname{Id}_{n n} & 0_{n n}
\end{array}\right) v\right) .
$$

It is easy to see that $\lambda^{\theta}$ is a Shalika functional for the representation $\pi^{\theta}$.

Theorem 4.9 [Friedberg and Jacquet 1993, Proposition 3.3].

$$
\gamma(s, \pi, \psi) L_{\lambda, v}^{\pi}(s)=L_{\lambda^{\theta}, v}^{\pi^{\theta}}(1-s) .
$$

Using this and Theorem 4.6 we obtain:

Corollary 4.10. There exists $N<0$ such that for any odd integer $N^{\prime} \leq-1$ and any polynomial $p$ on $\mathbb{C}$ there exists a seminorm $\beta$ on $V$ such that

$$
\left|p L_{\lambda, v}\right|_{N^{\prime}+N+\mathrm{iR}} \mid \leq \beta(v) .
$$

\section{Uniqueness of Shalika functionals}

Theorem 5.1. Let $(\pi, V)$ be an irreducible admissible representation of $G$. Let $\lambda$ be a Shalika functional. Then the functional $L(\lambda): V \rightarrow \mathbb{C}$ defined by

$$
L(\lambda)(v):=\frac{L_{\lambda, v}}{L_{\pi}}\left(\frac{1}{2}\right)
$$

is continuous.

Proof. By Theorem 4.6 we choose $M>1$ such that for any polynomial $p$ there exists a seminorm $\beta$ on $V$ such that $\left|p L_{\lambda, v}\right|_{M+\mathrm{i} \mathbb{R}} \mid \leq \beta(v)$. By Corollary 4.10 we choose $N<0$ such for any polynomial $p$ there exists a seminorm $\beta^{\prime}$ on $V$ such that $\left|p L_{\lambda, v}\right|_{N+\mathrm{iR}} \mid \leq \beta^{\prime}(v)$. Let $q$ be a polynomial such that the multiset of poles of $1 / q$ (with multiplicities) coincides with the multiset of poles of $\left.L_{\pi}\right|_{[N, M]+\mathrm{i} \mathbb{R}}$. 
Here, $[N, M]+\mathrm{i} \mathbb{R}$ denotes the strip $N \leq \operatorname{Re}(s) \leq M$. It is enough to show that the map $L^{\prime}(\lambda)$ defined by $L^{\prime}(\lambda)(v):=L_{\lambda, v} q(1 / 2)$ is continuous. Now there exists a seminorm $\alpha$ on $V$ such that, for any $v \in V$,

$$
\left|q L_{\lambda, v}\right|_{M+\mathrm{i} \mathbb{R}} \mid \leq \alpha(v) \quad \text { and } \quad\left|q L_{\lambda, v}\right|_{N+\mathrm{i} \mathbb{R}} \mid \leq \alpha(v) .
$$

By Theorem 4.1, for any $v \in V$, there exists $\Delta$ such that $\left|q L_{\lambda, v}(s)\right| \leq \alpha(v)$ if $s \in[N, M]+\mathrm{i} \mathbb{R}$ and $|\operatorname{Im} s| \geq \Delta$. Now by the maximal modulus principle $L^{\prime}(\lambda)(v) \leq$ $\alpha(v)$ for any $v \in V$.

Theorem 3.1 then implies:

Proposition 5.2. Let $(\pi, V)$ be an irreducible admissible representation of $G$. Then the map $L:\left(V^{*}\right)^{H U, \Psi} \rightarrow\left(V^{*}\right)^{H G_{1}}$ defined by

$$
L(\lambda)(v):=\frac{L_{\lambda, v}}{L_{\pi}}\left(\frac{1}{2}\right)
$$

is a monomorphism.

As mentioned in the introduction, the pair $\left(\mathrm{GL}_{2 n}, \mathrm{GL}_{n} \times \mathrm{GL}_{n}\right)$ is a Gelfand pair, by [Aizenbud and Gourevitch 2009, Theorem 8.2.4]; that is,

$$
\operatorname{dim}\left(V^{*}\right)^{\mathrm{GL}_{n}(F) \times \mathrm{GL}_{n}(F)} \leq 1 .
$$

Putting this together with the preceding proposition, we obtain the promised result:

Theorem 1.1. If $(\pi, V)$ is an irreducible admissible smooth Fréchet representation of $\mathrm{GL}_{2 n}(F)$, the space $\left(V^{*}\right)^{H U, \Psi}$ of Shalika functionals on $V$ has dimension at most 1 .

\section{Acknowledgements}

Aizenbud and Gourevitch thank Josef Bernstein, Wee Teck Gan and Binyong Sun for useful remarks.

\section{References}

[Aizenbud and Gourevitch 2008] A. Aizenbud and D. Gourevitch, "Schwartz functions on Nash manifolds”, Int. Math. Res. Not. (2008), issue 5, ID rnm 155. MR 2418286

[Aizenbud and Gourevitch 2009] A. Aizenbud and D. Gourevitch, "Generalized Harish-Chandra descent, Gelfand pairs, and an Archimedean analog of Jacquet-Rallis's theorem”, Duke Math. J. 149 (2009), 509-567.

[Casselman 1980] W. Casselman, "Jacquet modules for real reductive groups", pp. 557-563 in Proceedings of the International Congress of Mathematicians (Helsinki, 1978), Acad. Sci. Fennica, Helsinki, 1980. MR 83h:22025 Zbl 0425.22019

[Dixmier and Malliavin 1978] J. Dixmier and P. Malliavin, "Factorisations de fonctions et de vecteurs indéfiniment différentiables”, Bull. Sci. Math. 102:4 (1978), 307-330. MR 80f:22005 
[Friedberg and Jacquet 1993] S. Friedberg and H. Jacquet, "Linear periods", J. Reine Angew. Math. 443 (1993), 91-139. MR 94k:11058 Zbl 0782.11033

[Godement and Jacquet 1972] R. Godement and H. Jacquet, Zeta functions of simple algebras, Lecture Notes in Math. 260, Springer, Berlin, 1972. MR 49 \#7241 Zbl 0244.12011

[Jacquet and Rallis 1996] H. Jacquet and S. Rallis, "Uniqueness of linear periods", Compositio Math. 102:1 (1996), 65-123. MR 97k:22025 Zbl 0855.22018

[Wallach 1992] N. R. Wallach, Real reductive groups, II, Pure and Applied Mathematics 132, Academic Press, Boston, 1992. MR 93m:22018 Zbl 0785.22001

Received April 10, 2009.

AVRAHAM AIZENBUd

FACUlty of MATHEMATICS AND COMPUTER SCIENCE

WEIZMANN InSTITUTE OF SCIENCE

DEPARTMENT OF MATHEMATICS

POB 26

76100 REHOVOT

ISRAEL

aizenr@yahoo.com

http://www.wisdom.weizmann.ac.il/ aizenr/

DMITRY GOUREVITCH

FACUlTy OF MATHEMATICS AND COMPUTER SCIENCE

Weizmann Institute of SCIEnCE

DEPARTMENT OF MATHEMATICS

POB 26

76100 REHOVOT

ISRAEL

guredim@yahoo.com

http://www.wisdom.weizmann.ac.il/ dimagur/

HERVÉ JACQUET

COLUMBIA UNIVERSITY

DEPARTMENT OF MATHEMATICS

NEW YORK, NY 10027

UNITED STATES

hj@math.columbia.edu 